\title{
Root Furcation
}

National Cancer Institute

\section{Source}

National Cancer Institute. Root Furcation. NCI Thesaurus. Code C82059.

The region of a multirooted tooth at which the root divides. 